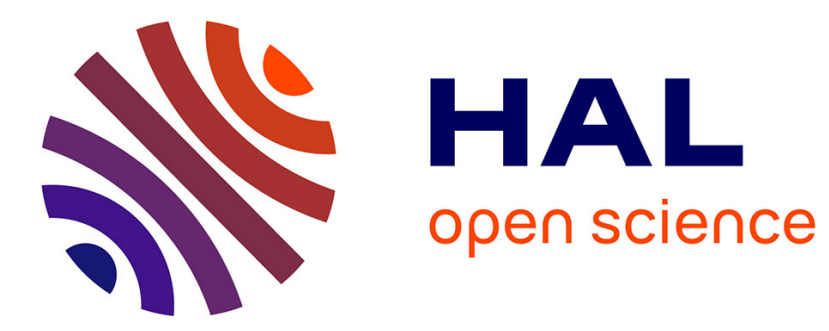

\title{
Spectrométrie IR pour la mesure de faibles variations d'émissivité
}

\author{
Ph. Pigeat, N. Pacia, B. Weber, D. Paulmier
}

\section{To cite this version:}

Ph. Pigeat, N. Pacia, B. Weber, D. Paulmier. Spectrométrie IR pour la mesure de faibles variations d'émissivité. Revue de Physique Appliquée, 1985, 20 (12), pp.863-868. 10.1051/rphysap:019850020012086300 . jpa-00245403

\section{HAL Id: jpa-00245403 https://hal.science/jpa-00245403}

Submitted on 1 Jan 1985

HAL is a multi-disciplinary open access archive for the deposit and dissemination of scientific research documents, whether they are published or not. The documents may come from teaching and research institutions in France or abroad, or from public or private research centers.
L'archive ouverte pluridisciplinaire HAL, est destinée au dépôt et à la diffusion de documents scientifiques de niveau recherche, publiés ou non, émanant des établissements d'enseignement et de recherche français ou étrangers, des laboratoires publics ou privés. 
Classification

Physics Abstracts

$07.65 \mathrm{G}$

\title{
Spectrométrie IR pour la mesure de faibles variations d'émissivité
}

\author{
Ph. Pigeat, N. Pacia, B. Weber et D. Paulmier \\ Laboratoire d'Etudes et de Recherches en Mécanique et Energétique des Surfaces $\left({ }^{+}\right)$, \\ 6, rue du Joli Cour, 54000 Nancy, France
}

(Reçu le 21 mars 1985, révisé le 3 juillet, accepté le 3 septembre 1985)

\begin{abstract}
Résumé. - Nous décrivons un spectromètre infrarouge double faisceau $(0,5 \mu \mathrm{m}-10 \mu \mathrm{m})$ adapté à l'étude de faibles variations d'émissivité monochromatique directionnelle d'une surface. Grâce à un système original de commutation des voies, on mesure sous vide (pression $10^{-7}$ torr), la différence d'énergie émise par les deux faces d'un ruban échantillon. C'est ainsi que nous avons mesuré la différence d'émissivité des deux faces d'un ruban de carbone lorsqu'un dépôt de platine est effectué sur l'une de celles-ci. Nous montrons qu'il est possible de mettre en évidence des variations d'émissivité de $0,3 \%$ avec une précision de $10 \%$. De telles variations sont engendrées par des dépôts de $3,6 \times 10^{15}$ atomes $/ \mathrm{cm}^{2}$ de platine déposés sur carbone.
\end{abstract}

\begin{abstract}
We describe here a double beam infrared spectrometer $(0.5-10 \mu \mathrm{m})$ well adapted for the measurement of small variation in spectral and directional emissivity of surface. With an original switch system, we measure under high vacuum $\left(10^{-7}\right.$ torr), the difference between the radiative power of each side of the sample. In this way, we have measured, the difference between the radiative power of each side of a carbon ribbon, when under vacuum we deposit platinum on one side. We show, that it is possible to measure $0.3 \%$ of variation of emissivity, with an accuracy of $10 \%$. Such a variation, is the result of a deposit of $3.6 \times 10^{15}$ atoms $/ \mathrm{cm}^{2}$ of platinum on the carbon surface.
\end{abstract}

\section{Introduction.}

Si les résultats sur les propriétés optiques des matériaux (réflexion, transmission, émissivité) sont nombreux, ils présentent néanmoins une dispersion telle qu'ils sont en fait souvent inutilisables [1]. Ceci vient du fait que pour chaque résultat énoncé dans la littérature, les caractéristiques physico-chimiques (rugosité, épaisseur du dépôt stoechiométrie, cristallographie, etc...) dont on sait qu'elles sont prépondérantes sont mal définies, sinon totalement ignorées. Des théories ou relations phénoménologiques qui traduiraient l'influence de ces paramètres sont rares et ce manque est particulièrement imporant lorsqu'il s'agit du pouvoir émissif des surfaces [2]. Ces théories nécessiteraient, pour leur élaboration et leur vérification, des résultats expérimentaux précis et où les influences de chaque paramètre puissent être décorrelées.

Pour étudier l'influence de ces paramètres sur l'émissivité d'une surface, nous avons choisi d'obser-

$\left(^{+}\right)$U.A. 865 du C.N.R.S. ver directement les variations d'énergie émise pour des températures usuellement rencontrées dans l'industrie $400<T<1500 \mathrm{~K}$ et donc sur un spectre qui s'étend de $\lambda=0,5 \mu \mathrm{m}$ à $\lambda=10 \mu \mathrm{m}$, ceci de manière directionnelle.

Pour atteindre la précision nécessaire à une telle étude, des conditions expérimentales particulières devaient être rassemblées :

- Grande sensibilité et minimisation des rayonnements ou absorptions parasites.

- Constance des conditions expérimentales pour chaque mesure.

- Maitrise des caractéristiques physico-chimiques des surfaces étudiées.

- Suppression de l'influence de la température durant l'observation de la modification d'émissivité $(\varepsilon)$ en fonction de la variation du paramètre étudié : or, aucun système expérimental proposé antérieurement ne satisfaisait toutes ces exigences, seul, un montage réalisé en 1980 [3] répondait correctement aux trois dernières conditions. Le principe de ce spectrophotomètre consiste à comparer les énergies émises par deux faces A et B d'un ruban échantillon 
ne présentant de différence que sur un paramètre. Ce ruban étant suffisamment fin, on s'affranchit ainsi du problème d'inégalité des températures entre les surfaces comparées, inégalité dont on sait qu'elle entraîne des erreurs très importantes sur les mesures d'émissivité [4]. Le résultat obtenu avec ce montage est de la forme $\varepsilon_{\mathrm{A}} / \varepsilon_{\mathrm{B}}$ (rapport des émissivités de chacune des faces). Nous avons conçu et réalisé un nouvel appareil qui reprend le principe général du précédent (comparaison de deux faces d'un même échantillon) mais où le circuit optique est conçu cette fois de manière à obtenir directement la différence d'émissivité $\left(\varepsilon_{\mathrm{A}}-\varepsilon_{\mathrm{B}}\right)=\Delta \varepsilon$, grâce à une mesure différentielle vraie. On montre que la sensibilité de ce nouvel appareil est telle que l'on peut détecter par exemple l'influence sur l'émissivité d'un dépôt de l'ordre de quelques couches atomiques (épaisseur $<4 \times 10^{15}$ at $\left./ \mathrm{cm}^{2}\right)$.

\section{Description de l'appareil (Fig. 1).}

Un double circuit focalise successivement sur la fente d'entrée d'un système de dispersion (prisme $\mathrm{NaCl}$ montage " Littrow ", muni d'un détecteur pyroélectrique Plessey PLT 222), les faisceaux d'énergie $E_{\mathrm{A}}$ et $E_{\mathrm{B}}$ émis par deux surfaces à comparer. Tout ce montage, dont les miroirs sont dorés face avant, est situé dans une enceinte identique à celle de l'appareil précédent (Fig. 1). Cette enceinte permet de travailler sous vide $\left(10^{-7}\right.$ torr) ou sous atmosphère contrôlée. La source est constituée d'une lame conductrice du matériau à étudier (ou support du matériau à étudier) chauffée par effet Joule. L'épaisseur de cette lame est suffisamment faible pour que la différence de température entre les deux faces soit négligeable, sa longueur suffisamment grande pour qu'il existe

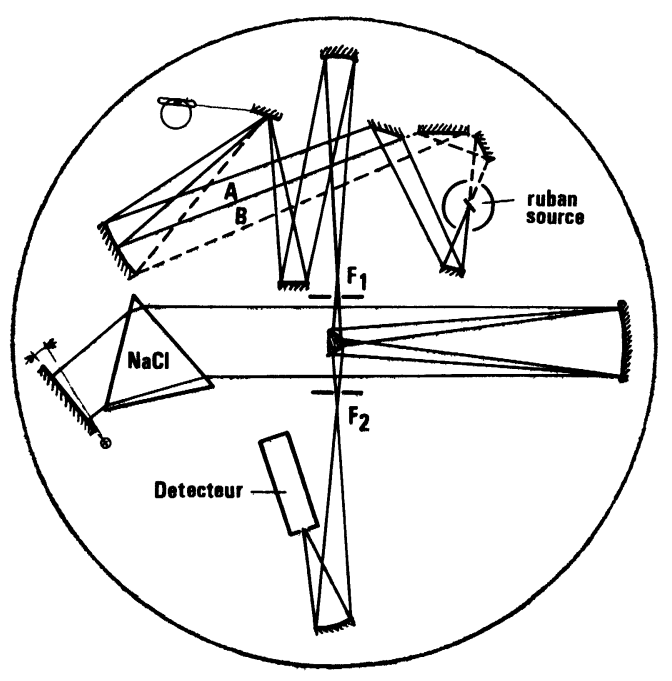

Fig. 1. - Schéma (vue de dessus) de l'enceinte et du spectrophotomètre qu'elle contient.

[Schematic view of the vacuum chamber and the spectrometer.] une zone isotherme plus large que la zone observée par le spectrophotomètre. Sur l'une de ces faces, un paramètre pourra être modifié. Ce paramètre restant inchangé, l'autre face, tout en subissant par ailleurs la même histoire thermique, chimique et mécanique, pourra jouer le rôle de référence. Ainsi, est-on sûr que seul interviendra dans la comparaison des énergies émises le paramètre étudié effectivement (rugosité, épaisseur d'une couche d'oxyde, etc...). Le support de cet échantillon (Fig. 2), placé à l'intérieur d'une double paroi refroidie par circulation

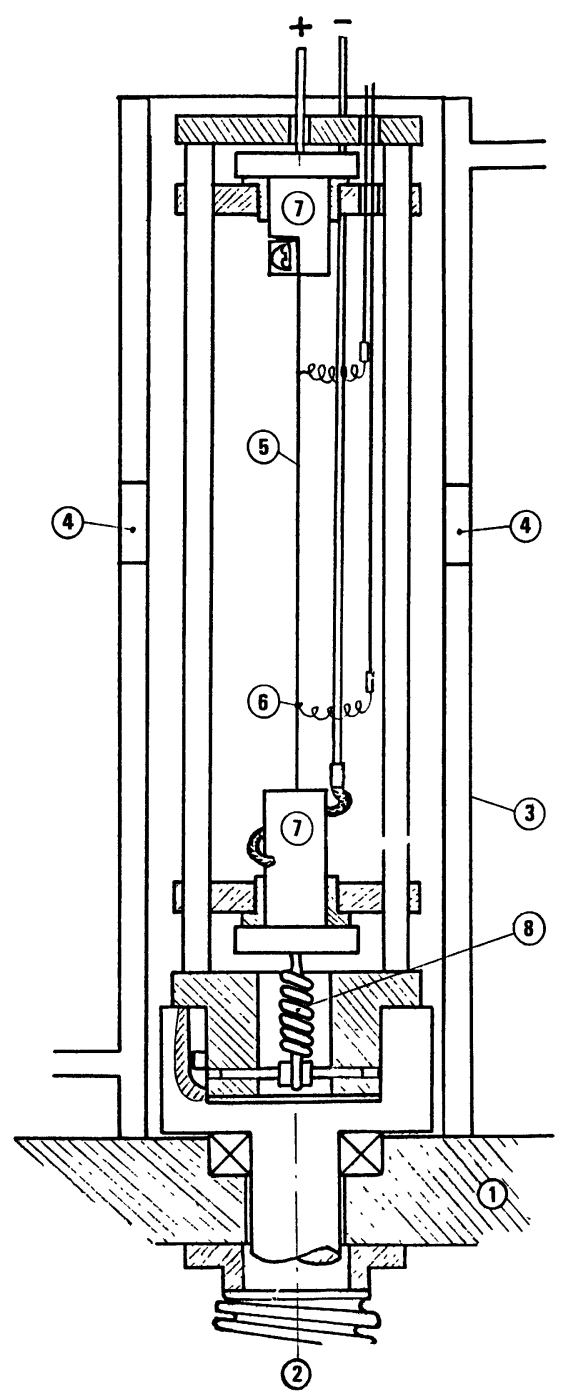

Fig. 2. - Schéma du support source : (1) Enceinte à vide. (2) Passage tournant (variation en $\theta$ ). (3) Double paroi absorbant le rayonnement parasite. (4) Ouverture symétrique (voie A et B). (5) Ruban échantillon. (6) Prises de potentiel. (7) Mords à écartement réglable. (8) Tension de l'échantillon (rattrapage de la dilatation).

[Specimen mounting:(1) Vacuum chamber. (2) Rotatory Motion Drive. (3) Double schielding absorbing stray radiation from the specimen. (4) Symmetric apertures for observation of the two beams emerging A and B. (5) Ribbon specimen. (6) Potential leads. (7) Mobile grips (for specimen position ajustement). (8) Spring (absorbing the specimen's thermal expansion).] 
d'eau qui élimine le rayonnement parasite, est monté de manière à ce que les voies $\mathrm{A}$ et $\mathrm{B}$ interceptent des faisceaux émis sous des angles d'émergence $\theta_{\mathbf{A}}$ et $\theta_{\mathbf{B}}$ identiques. La rotation de ce support permet la comparaison des indicatrices d'émission de chacune des faces en conservant l'égalité $\theta_{\mathrm{A}}=\theta_{\mathrm{B}}$.

Sur l'appareil réalisé précédemment [2], la comparaison des énergies $E_{\mathrm{A}}$ et $E_{\mathrm{B}}$ émises par les faces $\mathrm{A}$ et $\mathrm{B}$ de l'échantillon se fait par l'intermédiaire de l'énergie émise par les pales d'un obturateur à température ambiante. La méthode d'analyse du signal aboutit au résultat $\left(\varepsilon_{\mathbf{A}} / \varepsilon_{\mathbf{B}}\right)_{\lambda \theta}$. Pour obtenir ce résultat, un modulateur commun aux deux voies, un miroir tournant de commutation, un amplificateur à détection synchrone, ainsi qu'un montage électronique adapté sont nécessaires. Dans le cas présent, nous avons pu gagner en sensibilité et obtenir directement la différence d'émissivité $\Delta \varepsilon$ entre les deux faces de l'échantillon en utilisant la propriété du détecteur pyroélectrique d'être différentiel (sensible à des différences d'énergies incidentes). Grâce à un système simple et original de modulation sinusoïdale entre ces énergies $E_{\mathrm{A}}$ et $E_{\mathrm{B}}$ incidentes, on s'affranchit du filtrage parasite des hautes fréquences dans l'électronique d'amplification.

\section{Principe des mesures (commutation sinusoïdale).}

Les modulateurs ou commutateurs existant et décrits dans la littérature [5-7] (modulateur exempt de transmission) sont toujours du type miroir oscillant ou miroir tournant (Fig. 3). Lorsque les sources sont éloignées, le passage du signal de l'une au signal de l'autre est en général discontinu. On peut imaginer le rendre sinusoïdal, à l'aide de diaphragmes de formes particulières, difficiles à régler (exemple Fig. 3) mais dans ce cas la forme du faisceau est déterminée et ne peut être modifiée.

Le système que nous proposons, permet d'obtenir un passage continu du signal A au signal B de forme quelconque et sinusoïdal en particulier [8].
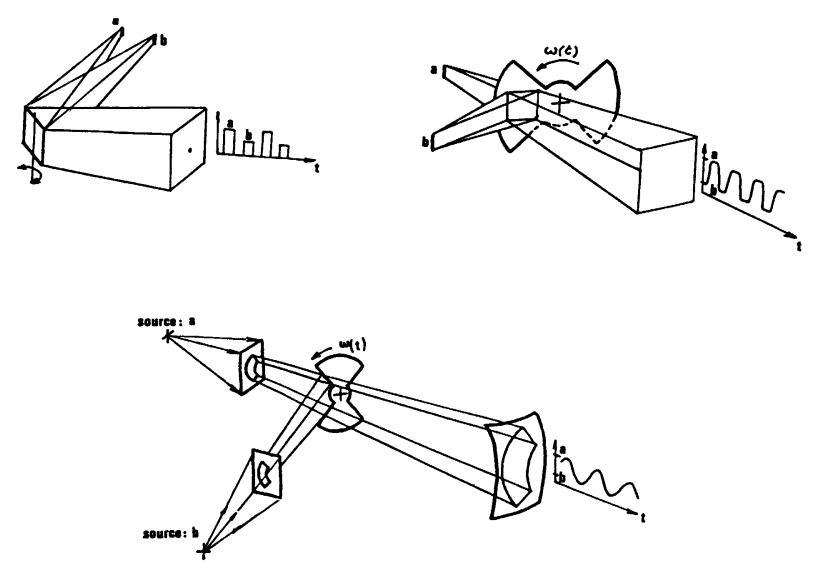

Fig. 3. - Exemple de commutateur.

[Example of standard switch.]

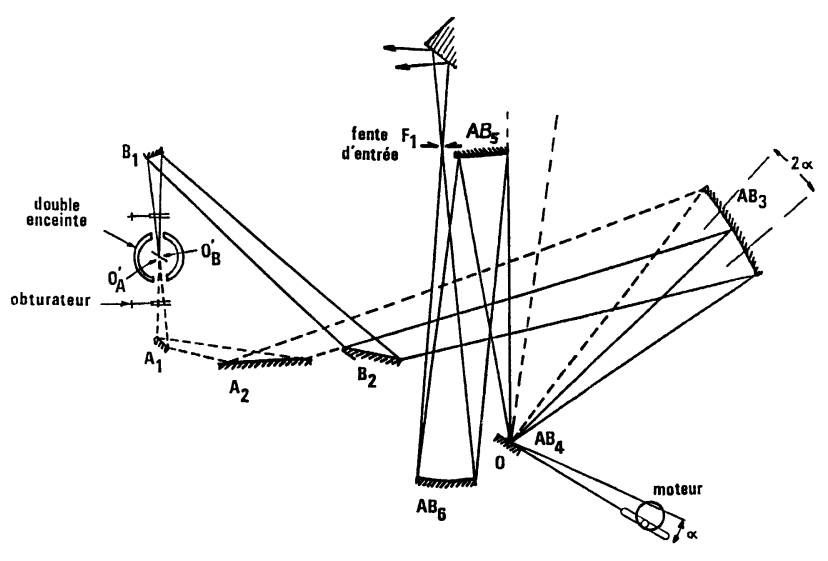

Fig. 4. - Schéma des circuits optiques du double faisceau et du système de commutation sinusoïdale.

[Schematic view of the double beams and of the sinusoïdal switch device.]

L'angle solide analysé par le système de dispersion issu de la fente est refocalisé par le miroir concave $A_{6}$ sur le miroir plan $A_{3}$ en 0 , ceci par l'intermédiaire du miroir plan $\mathrm{AB}_{5}$ (Fig. 4). Le miroir $\mathrm{AB}_{4}$ oscille autour d'un axe contenu dans le plan réflecteur, cet axe de rotation est le grand axe de l'image de $F$ sur $A B_{4}$. Le point $O$ étant le point source du miroir concave $A_{3}$, le faisceau divergent issu de $A B_{4}$ balaie $A_{3}$, et est refocalisé au point fictif $0^{\prime}$. L'angle solide issu de $\mathrm{AB}_{3}$ est divisé en deux parties égales (A et $B$ ) grâce au miroir plan-couteau $B_{2}$ qui, par l'intermédiaire de $B_{1}$ emmène la moitié $B$ en $O_{B}^{\prime}$ sur la face $B$ de l'échantillon. Le miroir plan $A_{2}$ guide par l'intermédiaire de $A_{1}$ la seconde moitié du faisceau vers $\mathrm{O}_{\mathrm{A}}^{\prime}$ sur la face $\mathrm{A}$ de l'échantillon. Pour des dimensions du circuit optique adéquates, le faisceau analysé par le système de dispersion peut, suivant les positions extrêmes du balayage de $\mathbf{A B}_{4}$, être issu de $\mathrm{O}_{\mathrm{A}}^{\prime}$ ou de $\mathrm{O}_{\mathbf{B}}^{\prime}$. En effet, selon l'inclinaison de $\mathbf{A B}_{4}$, les faisceaux contigus $A$ et $B$ issus de $A B_{3}$, seront interceptés dans des proportions différentes, pour être ensuite focalisés sur la fente d'entrée $F$. Sur la figure 5 , on a schématisé l'interception par $\mathrm{AB}_{5}$ des faisceaux $A$ et $\mathrm{B}$ pour une oscillation sinusoïdale de $\mathrm{AB}_{4}$ dont l'amplitude est ajustée pour que le plan médian du faisceau $\mathrm{AB}$ balaie le miroir $\mathrm{AB}_{5}$ de largeur $l$ (Fig. 5). On aura un faisceau focalisé sur $F$ dont l'énergie sera fonction de celle des faisceaux issus des faces A et $\mathrm{B}\left(E_{\mathrm{A}}\right.$ et $\left.E_{\mathrm{B}}\right)$ selon l'équation :

$$
E_{\text {entrant }}=\frac{l}{2}\left[E_{\mathrm{A}}(1+\cos \omega t)+E_{\mathrm{B}}(1-\cos \omega t)\right] .
$$

Pour obtenir l'oscillation de $\mathrm{AB}_{4}$ plusieurs montages connus pouvaient être envisagés suivant la forme du signal désiré. Le montage retenu pour sa simplicité et son faible coût est un système "bielle manivelle " (schématisé Fig. 4). Ce montage est facile à régler puisque le déplacement en $X, Y$ du support moteur suffit au réglage à la fois de la direction et de l'ampli- 


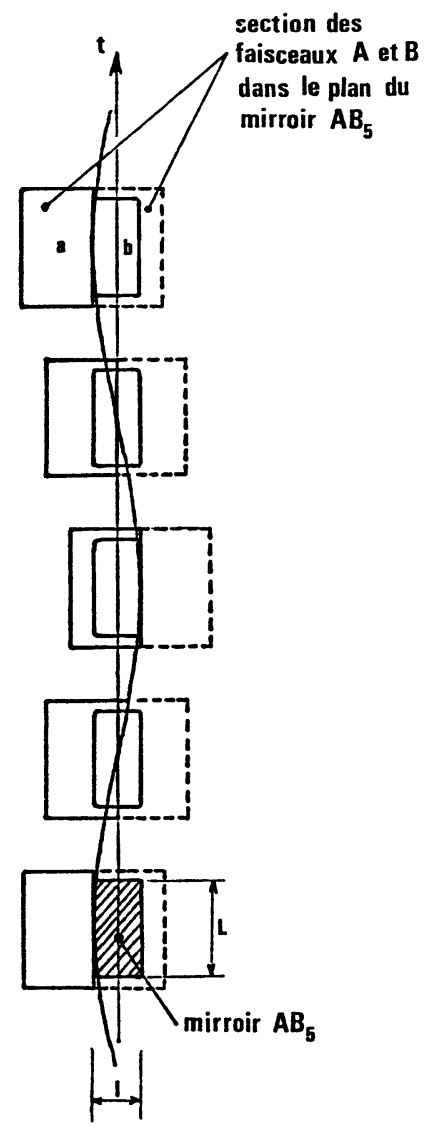

Fig. 5. - Déplacement dans le plan du miroir $\mathrm{AB}_{5}$ des sections (a) et (b) des faisceaux contigus $A$ et $B$ durant l'oscillation sinusoïdale du miroir $\mathrm{AB}_{4}$, l'amplitude de cette oscillation étant ajustée pour que la frontière entre (a) et (b) balaie la largeur $l$ du miroir $\mathbf{A B}_{5}$.

[Scanning (in the plan of the mirror $\mathrm{AB}_{5}$ ) of sections (a) and (b) of adjacent beams $A$ and $B$ during the sinusoidal oscillation of the mirror $\mathbf{A B}_{4}$. In this configuration, the amplitude is ajusted in order that the median plan between (a) and (b) scan the width of the mirror $\left.\mathrm{AB}_{5}.\right]$

tude du balayage de $\mathrm{AB}$. Le signal de référence pour la détection synchrone est obtenu par la modulation d'une photodiode directement sur le bloc moteur donc loin du circuit optique proprement dit.

Ce circuit optique a été conçu pour minimiser les différences entre les voies : même longueur de trajet optique, même nombre de réflexions, même polarisation du rayonnement. L'emplacement des miroirs $A_{1}$ et $B_{1}$ alignés avec l'axe de rotation du ruban source a pour conséquence que, quel que soit l'orientation du ruban, les angles d'émergence des rayonnements analysés sont rigoureusement identiques $\left(\theta_{\mathbf{A}}=\theta_{\mathrm{B}}\right)$ durant l'étude des indicatrices d'émission. Deux obturateurs, conçus de manière à rester à température ambiante et manipulables de l'extérieur de l'enceinte peuvent venir couper les voies A ou B, (ou protéger les miroirs $A_{1}$ et $B_{1}$ durant certains traitements de l'échantillon (oxydation, déposition, vaporisation, nettoyage...)).
Pour une position du miroir de Littrow ( $\lambda$ fixée) et un angle d'émergence donné $\theta_{\mathrm{A}}=\theta_{\mathrm{B}}=\theta$, les obturateurs ouverts, le détecteur pyroélectrique reçoit un faisceau dont l'énergie varie sinusoïdalement de $E_{\mathrm{A}}$ à $E_{\mathrm{B}}$. On montre alors que le signal de sortie du détecteur est aussi sinusoïdal [9] d'amplitude proportionnelle à $E_{\mathrm{A}}-E_{\mathrm{B}}$. Un amplificateur à détection synchrone extrait alors un résultat de la forme :

soit :

$$
E_{\lambda \theta}=K\left(E_{\mathrm{A}_{\lambda \theta}}-E_{\mathrm{B}_{\lambda \theta}}\right)
$$

$$
E_{\lambda \theta}=K\left(\alpha \varepsilon_{\mathbf{A}_{\lambda \theta}} \mathfrak{L}_{T_{\mathbf{A}_{\lambda \theta}}}-\beta \varepsilon_{\mathbf{B}_{\lambda \theta}} \mathfrak{L}_{T_{\mathbf{B}_{\lambda \theta}}}\right)
$$

l'égalité des températures des faces $\mathrm{A}$ et $\mathrm{B}$ implique :

avec :

$$
E_{\lambda \theta}=K\left(\alpha \varepsilon_{\mathbf{A}_{\lambda \theta}}-\beta \varepsilon_{\mathbf{B}_{\lambda \theta}}\right) \mathcal{L}_{T_{\lambda \theta}}
$$

$-\mathfrak{L}_{T \lambda \theta}$ : la luminance du corps noir à la température $T$ pour la longueur d'onde $\lambda$ sous l'incidence $\theta$;

- $K$ : facteur lié au calibra des appareils de mesure;

$-\varepsilon_{\mathrm{A} \lambda \theta}$ : l'émissivité de la face $\mathrm{A}$ à la longueur d'onde $\lambda$ et sous l'incidence $\theta$;

$-\alpha, \beta$, les facteurs de transmission des voies A et $\mathrm{B}$. Les facteurs de transmission $(\alpha, \beta)$ sont proches de l'unité et très peu différents par conception du double circuit, d'où :

$$
E_{\lambda \theta}=K \alpha \mathcal{L}_{T_{\lambda \theta}}\left(\varepsilon_{\mathrm{A} \lambda \theta}-\varepsilon_{\mathbf{B}_{\lambda \theta}}\right) .
$$

Si des dépôts parasites, sur les miroirs $A_{1}$ et $B_{1}$, par exemple, viennent à modifier ce réglage, ces facteurs seront obtenus par l'étalonnage de l'appareil sur un corps noir conçu spécialement (Fig. 6). Ce

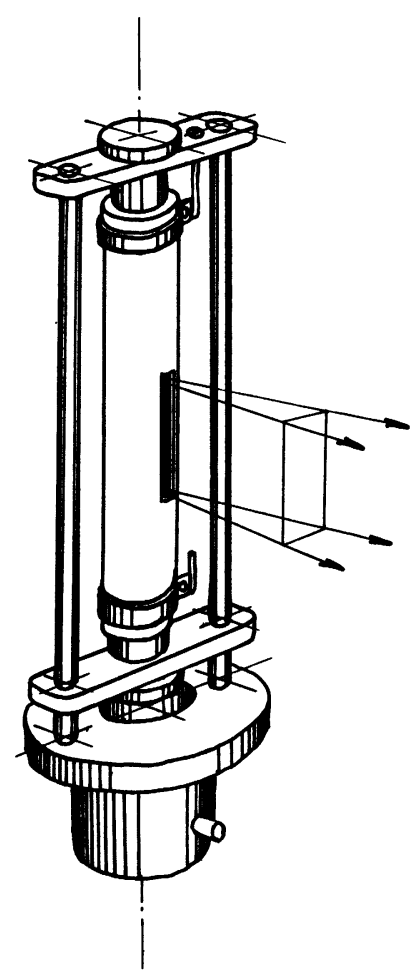

Fig. 6. - Schéma du montage du corps noir d'étalonnage.

[Mounting of the calibrating black body.] 
corps noir est constitué d'un tube « Pirox » (chromite de lanthane) placé sur le support échantillon et chauffé par effet Joule, qui présente une ouverture longitudinale de surface équivalente à la surface analysée sur les rubans échantillons.

On montre ainsi en (4), la surface $A$ étant la surface de référence, que l'on obtient donc directement l'évolution de $\left(\varepsilon_{\mathrm{A}}-\varepsilon_{\mathrm{B}}\right)$ en fonction de l'évolution du paramètre à étudier.

Ces résultats peuvent alors être mis sous la forme qui nous intéresse, à savoir :

$$
\frac{\Delta\left(\varepsilon_{\mathrm{A}}-\varepsilon_{\mathrm{B}}\right)}{\varepsilon_{\mathrm{A}}-\varepsilon_{\mathrm{B}}}=\frac{\Delta \varepsilon_{\mathrm{B}}}{\varepsilon_{\mathrm{B}}\left(\frac{\varepsilon_{\mathrm{A}}}{\varepsilon_{\mathrm{B}}}-1\right)}=k \frac{\Delta \varepsilon_{\mathrm{B}}}{\varepsilon_{\mathrm{B}}}
$$

Sous certaines conditions, il est possible d'obtenir directement la variation relative de $\varepsilon_{\mathbf{B}}$ sans se soucier de $\alpha, \beta, k$ et $\mathcal{L}_{T}$. C'est en particulier le cas, lorsque la voie A est obturée et que l'énergie émise par cet obturateur (qui est à température ambiante, soit $300 \mathrm{~K}$ ) est négligeable devant l'énergie émise par la surface étudiée. L'échantillon étudié étant à $900 \mathrm{~K}$ l'erreur commise en négligeant l'énergie émise par l'obturateur pour $\lambda=3 \mu \mathrm{m}$ est inférieure à $10^{-5}$. Cette approximation permet d'écrire :

$$
E=\left(\beta \varepsilon_{\mathrm{B}} \mathcal{L}_{T}-\alpha \varepsilon_{\mathrm{obt}} \mathcal{L}_{300 \mathrm{~K}}\right) \simeq \beta \varepsilon_{\mathrm{B}} \mathcal{L}_{T}
$$

pour aboutir au rapport qui nous intéresse :

$$
\frac{\beta\left(\varepsilon_{\mathrm{B}}+\Delta \varepsilon_{\mathrm{B}}\right) \mathcal{L}_{T}-\beta \varepsilon_{\beta} \mathcal{L}_{T}}{\beta \varepsilon_{\mathrm{B}} \mathcal{L}_{T}}=\frac{\Delta \varepsilon_{\mathrm{B}}}{\varepsilon_{\mathrm{B}}} .
$$

La mesure (4) peut être enregistrée en fonction de $\theta$ (indicatrice d'émission) ou en fonction de la longueur d'onde $\lambda$ (ceci de 0,5 à $10 \mu \mathrm{m}$ ) soit sur une table traçante, soit sur un microordinateur (HP 85) qui effectue automatiquement le calcul de $\Delta \varepsilon_{\mathrm{B}} / \varepsilon_{\mathrm{B}}$ (Eq. (5)). La rotation du miroir de Littrow est commandée par un motoréducteur; son balayage linéaire dans le temps est traduit en tension. Le défilement en longueur d'onde qu'il provoque n'est quant à lui pas linéaire (propriété du prisme). Il est donc nécessaire d'utiliser une conversion tension-longueur d'onde obtenue lors de l'étalonnage de l'appareil grâce à l'utilisation de filtres interférentiels.

\section{Evaluation de la sensibilité de l'appareil.}

L'évolution de l'émissivité d'une surface de carbone au cours d'une déposition de platine par condensation sous vide, est l'exemple de mesure réalisé sur notre appareil que nous exposerons ici, pour illustrer la sensibilité de la méthode expérimentale que nous venons de décrire.

Parallèlement à un ruban de carbone [" Papiex "; $L=180 \mathrm{~mm}, l=10 \mathrm{~mm}$, épaisseur $100 \mu \mathrm{m}]$ on a placé à $12 \mathrm{~mm}$, un ruban de platine de longueur telle que la zone de carbone observée optiquement, soit largement contenue dans la zone recouverte par le dépôt de platine. Les faces $\mathbf{A}$ et $\mathbf{B}$ du ruban de carbone sont observées sous une incidence de $45^{\circ}$, pour ne pas être gêné par la présence du ruban évaporateur. La voie A observe la face du ruban de carbone sans dépôt qui joue le rôle de surface de référence; la voie $B$ observe la face sur laquelle se fait le dépôt de platine Excepté la présence de ce dépôt (forme ou épaisseur) les autres paramètres sont communs aux deux surfaces et n'interviendront pas dans la mesure.

Pour conserver aux échantillons étudiés sur notre appareil, une température constante durant l'expérience, (la connaissance de la valeur absolue de cette température n'étant pas prépondérante) deux techniques peuvent être utilisées :

- une stabilisation du courant d'alimentation de l'échantillon à partir de la résistance de la zone isotherme [2], la comparaison de cette résistance $R(T)$ à la résistance à froid $R(300 \mathrm{~K})$ donnant grâce à la courbe résistivité-température du matériaux, la valeur de $T$;

- une stabilisation du courant d'alimentation à partir de l'énergie émise de la surface de référence à une longueur d'onde $\lambda$ fixée; très précise cette méthode de pyrométrie différentielle permet de stabiliser la température de $900 \mathrm{~K}$ avec une précision supérieure au millième.

C'est cette dernière technique que nous avons utilisé pour stabiliser la température des rubans de carbone étudiés ici. Après recuit de ces rubans à $1500 \mathrm{~K}$ pendans $3 \mathrm{~h}$, la déposition est effectuée par période durant lesquelles le ruban de carbone est éteint et le ruban de platine porté à $1600 \mathrm{~K}$. Les obturateurs sont alors fermés et protègent les miroirs $A_{1}$ et $B_{1}$. Entre ces périodes de déposition, le ruban de platine est éteint et le ruban de carbone est porté à la température $T$ à laquelle se fait l'observation spectrale. De cette manière, nous avons pu suivre la différence d'émissivité entraînée par la présence du platine sur le carbone en fonction du temps de déposition donc de la quantité de platine déposée. Les résultats reportés sur la figure 7 ont été obtenus à la température d'émission de $900 \mathrm{~K}$ et pour la longueur d'onde $\lambda=3 \mu \mathrm{m}$. Pour des expositions au platine importantes on constate que le signal ne varie plus. Nous considérons alors qu'il est représentatif de celui qu'émettrait une surface de platine massif. Pour ces dépôts épais à $T=900 \mathrm{~K}$ et $\lambda=3 \mu \mathrm{m}$ la différence d'énergie enregistrée est $1,63 \times 10^{-6} \mathrm{~W}$, alors que l'énergie mesurée issue de la surface de carbone de référence est restée constante et égale à $2,5 \times 10^{-6} \mathrm{~W}$. Le rapport $\varepsilon_{\mathrm{pt}} / \varepsilon_{\mathrm{c}}$ obtenu, est compatible avec les rapports calculés à partir des résultats $\varepsilon_{\mathrm{pt}}$ et $\varepsilon_{\mathrm{c}}$ mentionnés dans la littérature, si l'on prend soin de comparer des émissivités du carbone et du platine à forte rugosité [1].

Pour des quantités de platine déposées très faibles, on constate que la face recouverte de platine émet plus que la face de carbone. En effet $\varepsilon_{\mathrm{pt}}-\varepsilon_{\mathrm{c}}$ est 


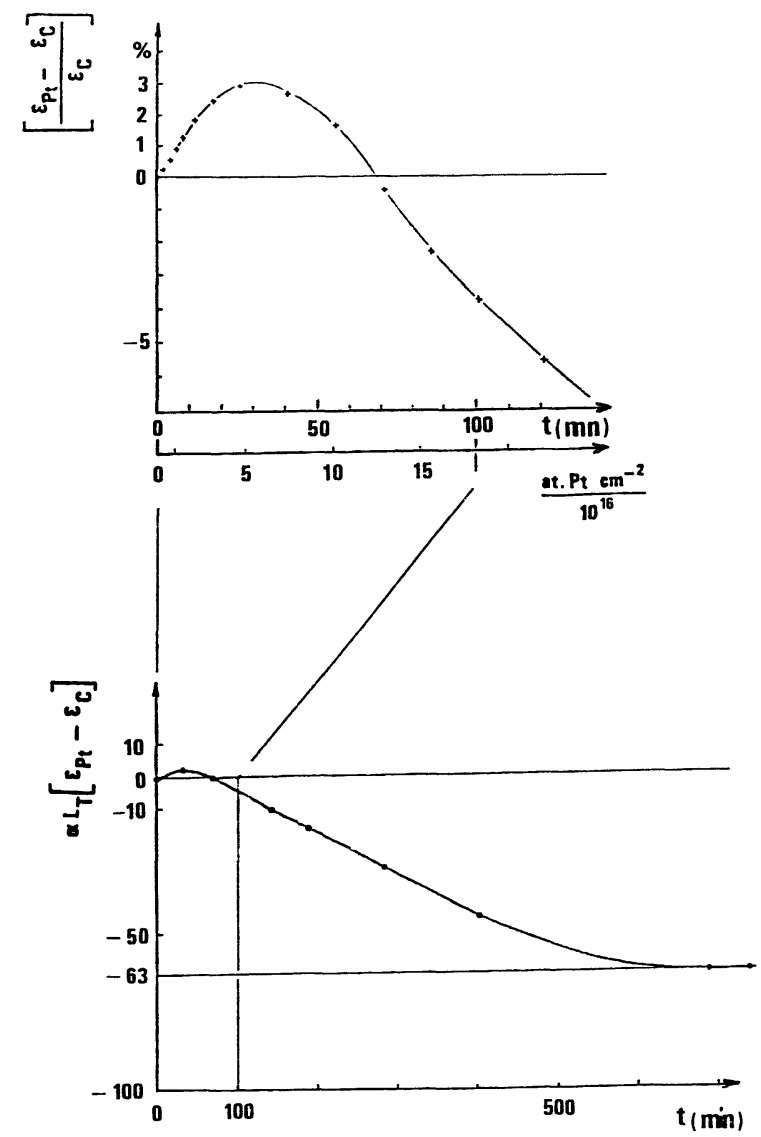

Fig. 7. - Evolution de la différence des énergies émises par les faces carbone et carbone + platine à $\theta=0, \lambda=3 \mu \mathrm{m}$, $T=900 \mathrm{~K}$, en fonction du temps de déposition du platine.

[Variation of the difference between energies emitted of the carbon surface and carbon + platinum surface at $\theta=0$; $\lambda=3 \mu \mathrm{m}, T=900 \mathrm{~K}$ during the platinum deposition.]

alors positif. La valeur maximale de cette augmentation est obtenue pour un dépôt de $5,49 \times 10^{16} \mathrm{at} / \mathrm{cm}^{2}$ et vaut $3 \%$. Ce maximum dans la variation d'émissivité en fonction de la quantité de platine déposée n'est pas surprenant dans la mesure où les épaisseurs mises en jeu sont alors de l'ordre de la centaine d'Angstroms et que dans ce cas l'indice complexe du dépôt est très différent de celui du matériau massif [11].
Pour interpréter ce résultat il serait nécessaire d'accumuler un grand nombre d'autres mesures d'émissivité faites dans des conditions différentes, mais aussi et surtout des résultats obtenus par d'autres techniques d'analyse des surfaces. Néanmoins, nous montrons clairement que notre appareil est capable de mesurer de très faibles variations d'émissivité. A la température de $900 \mathrm{~K}$ et à la longueur d'onde $\lambda=3 \mu \mathrm{m}$, notre appareil détecte une augmentation de l'énergie émise de $0,75 \times 10^{-8} \mathrm{~W}$ pour un dépôt de $3,6 \times 10^{15}$ at $/ \mathrm{cm}^{2}$ de platine. Comparé à $2,5 \times 10^{-6} \mathrm{~W}$ émis par la surface de carbone, l'équivalent en watt du bruit de fond de la chaîne de mesure étant inférieur à $10^{-9} \mathrm{~W}$, on montre que l'appareil est capable d'observer des variations d'émissivité de $0,3 \%$ avec une précision de $10 \%$.

\section{Conclusions.}

Nous venons de décrire un nouveau spectrophotomètre infrarouge double faisceau particulièrement bien adapté aux mesures différentielles de très faibles variations d'émissivité monochromatiques et directionnelles. Ce spectromètre sous vide, grâce notamment à un système original de commutation sinusoïdale des voies, offre une sensibilité telle qu'il ouvre de nouvelles perspectives quant à l'étude ou à la caractérisation des surfaces par le rayonnement thermique. On montre que cet appareil est capable de séparer et mesurer de très faibles variations d'émissivité engendrées par la modification d'un paramètre de surface sur l'exemple mentionné : l'influence sur $\varepsilon_{\lambda \theta}$ du carbone du dépôt de quelques $10^{15}$ atomes de platine par $\mathrm{cm}^{2}$.

Ce type de mesure en liaison avec une bonne caractérisation du paramètre choisi (on peut envisager outre les dépôts : la rugosité, le dopage, l'oxydation etc...) peut apporter des résultats précis, nécessaires à la mise au point des modèles exigés dans des domaines aussi importants que la pyrométrie, la thermographie, ou même l'analyse non destructive des surfaces.

\section{Bibliographie}

[1] Touloukian, Y. S., DewITT, D. P., Thermophysical Properties of Matter (Plenum, N.Y.) 7 (1970).

[2] Pigeat, P., Thèse, Nancy (1980).

[3] Pigeat, P., Paulmier, D., Revue Phys. Appl. 17 (1982) 393.

[4] Herve, P., Thèse, Paris (1977).

[5] Dolin, S. A., Kruegle, M. A., Penzias, G. J., Appl. Optics 6 (1967) 267.

[6] STEwarT, J. E., Infrared Spectroscopy Experimental Methods and Techniques (M. Dekker Inc., N.Y.) 1970.
[7] Theis, D., Busse, W., J. Phys. E Sci. Instrum. 10 (1977) 57.

[8] Furtak, T. E., Appl. Optics 16 (1977) 803.

[9] Pigeat, P., Dossier Technique ANVAR (1984).

[10] Hadni, A., J. Physique 24 (1963) 694.

[11] Heavens, O. S., Optical properties of thin solid films (Butterworths Sci. Pub., London) 1955. 